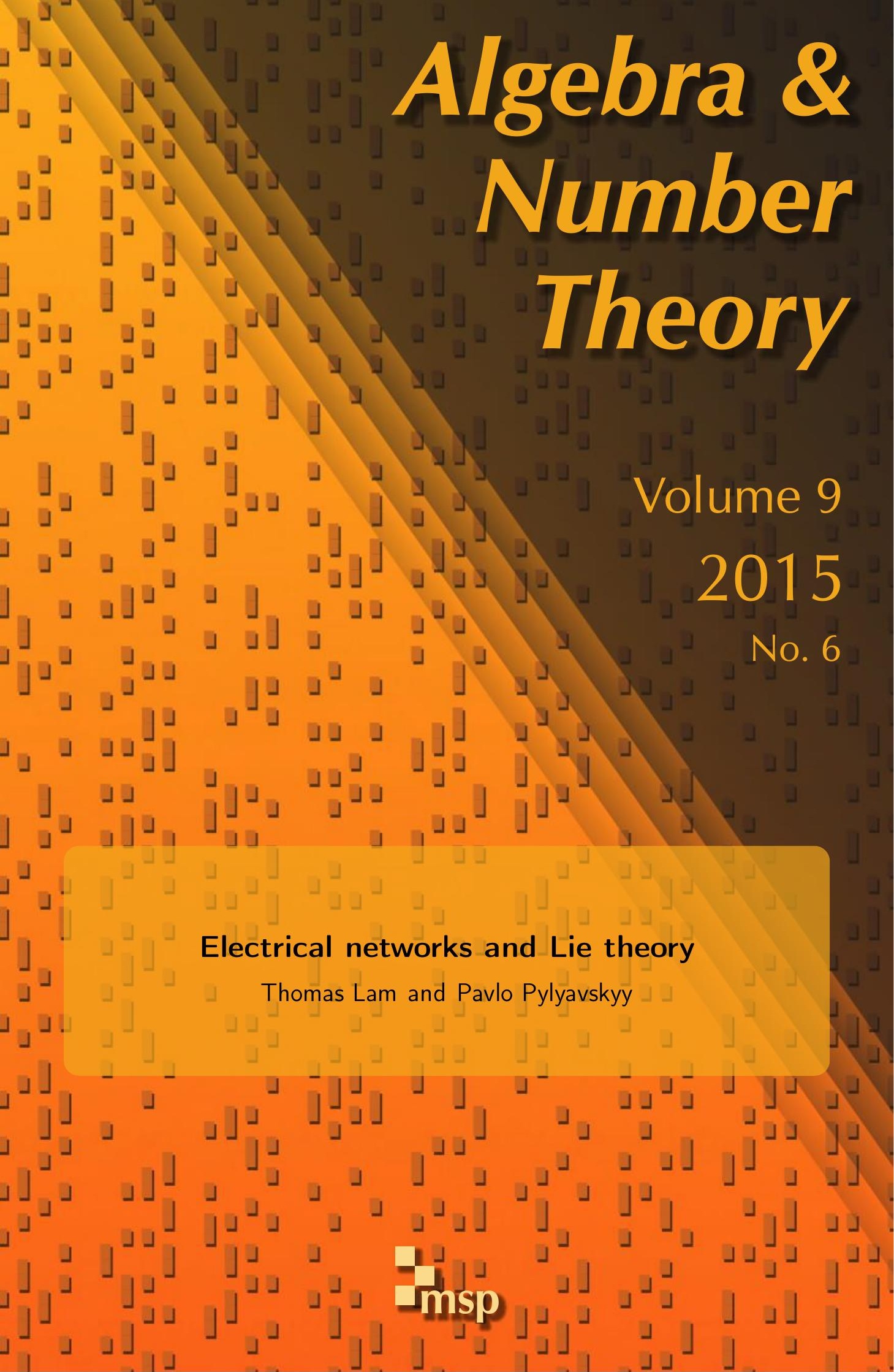




\title{
Electrical networks and Lie theory
}

\author{
Thomas Lam and Pavlo Pylyavskyy
}

\begin{abstract}
We introduce a new class of "electrical” Lie groups. These Lie groups, or more precisely their nonnegative parts, act on the space of planar electrical networks via combinatorial operations previously studied by Curtis, Ingerman and Morrow. The corresponding electrical Lie algebras are obtained by deforming the Serre relations of a semisimple Lie algebra in a way suggested by the star-triangle transformation of electrical networks. Rather surprisingly, we show that the type $A$ electrical Lie group is isomorphic to the symplectic group. The electrically nonnegative part $\left(\mathrm{EL}_{2 n}\right)_{\geq 0}$ of the electrical Lie group is an analogue of the totally nonnegative subsemigroup $\left(U_{n}\right)_{\geq 0}$ of the unipotent subgroup of $\mathrm{SL}_{n}$. We establish decomposition and parametrization results for $\left(\mathrm{EL}_{2 n}\right)_{\geq 0}$, paralleling Lusztig's work in total nonnegativity, and work of Curtis, Ingerman and Morrow and of Colin de Verdière, Gitler and Vertigan for networks. Finally, we suggest a generalization of electrical Lie algebras to all Dynkin types.
\end{abstract}

\section{Introduction}

We consider the simplest of electrical networks, namely those that consist of only resistors. The electrical properties of such a network $N$ are completely described by the response matrix $L(N)$, which computes the current that flows through the network when certain voltages are fixed at the boundary vertices of $N$. The study of the response matrices of planar electrical networks has led to a robust theory; see Curtis, Ingerman and Morrow [CIM 1998], or Colin de Verdière, Gitler and Vertigan [CdVGV 1996]. Kennelly [1899] described a local transformation (see Figure 3) of a network, called the star-triangle or $Y-\Delta$ transformation, which preserves the response matrix of a network. This transformation is one of the many places where a Yang-Baxter-style transformation occurs in mathematics or physics.

Curtis, Ingerman and Morrow [CIM 1998] studied the operations of adjoining a boundary spike and adjoining a boundary edge to (planar) electrical networks. Our point of departure is to consider these operations as one-parameter subgroups of a

Lam was partially supported by NSF grant DMS-0901111, and by a Sloan Fellowship. Pylyavskyy was partially supported by NSF grant DMS-0757165.

MSC2010: 05E15.

Keywords: Lie algebras, Serre relations, electrical networks. 
Lie group action. The star-triangle transformation then leads to an "electrical Serre relation" in the corresponding Lie algebra, which turns out to be a deformation of the Chevalley-Serre relation for $\mathfrak{s l}_{n}$ :

Serre relation: $\left[e,\left[e, e^{\prime}\right]\right]=0$; electrical Serre relation: $\left[e,\left[e, e^{\prime}\right]\right]=-2 e$.

The corresponding one-parameter subgroups satisfy a Yang-Baxter style relation which is a deformation of Lusztig's relation in total positivity:

Lusztig's relation:

$$
u_{i}(a) u_{j}(b) u_{i}(c)=u_{j}\left(\frac{b c}{a+c}\right) u_{i}(a+c) u_{j}\left(\frac{a b}{a+c}\right) ;
$$

electrical relation:

$$
u_{i}(a) u_{j}(b) u_{i}(c)=u_{j}\left(\frac{b c}{a+c+a b c}\right) u_{i}(a+c+a b c) u_{j}\left(\frac{a b}{a+c+a b c}\right) .
$$

We study in detail the Lie algebra $\mathfrak{e l}_{2 n}$ with $2 n$ generators satisfying the electrical Serre relation. An (electrically) nonnegative part $\left(\mathrm{EL}_{2 n}\right)_{\geq 0}$ of the corresponding Lie group $\mathrm{EL}_{2 n}$ acts on planar electrical networks with $n+1$ boundary vertices, and one obtains a dense subset of all response matrices of planar networks in this way. This nonnegative part is a rather precise analogue of the totally nonnegative subsemigroup $\left(U_{2 n+1}\right)_{\geq 0}$ of the unipotent subgroup of $\mathrm{SL}_{2 n+1}$, studied in Lie-theoretic terms by Lusztig [1994]. We show (Proposition 4.2) that $\left(\mathrm{EL}_{2 n}\right)_{\geq 0}$ has a cell decomposition labeled by permutations $w \in S_{2 n+1}$, precisely paralleling one of Lusztig's results for $\left(U_{2 n}\right)_{\geq 0}$ and reminiscent of the Bruhat decomposition. This can be considered an algebraic analogue of parametrization results in the theory of electrical networks [CIM 1998; CdVGV 1996]. Surprisingly, $\mathrm{EL}_{2 n}$ itself is isomorphic to the symplectic Lie group $\mathrm{Sp}_{2 n}(\mathbb{R})$ (Theorem 3.1). This semisimplicity does not in general hold for electrical Lie groups: $\mathrm{EL}_{2 n+1}$ is not semisimple. We also describe (Theorem 4.10) the stabilizer Lie algebra of the infinitesimal action of $\mathfrak{e l}_{2 n}$ on electrical networks. We caution that the nonnegative part $\left(\mathrm{EL}_{2 n}\right)_{\geq 0}$ is distinct from Lusztig's totally nonnegative part $\operatorname{Sp}_{2 n}(\mathbb{R})_{\geq 0}$ of the symplectic group.

While we focus on the planar case in this paper, we shall connect the results of this paper to the inverse problem for electrical networks on cylinders in a future paper. There we borrow ideas from representation theory, such as those of crystals and $R$-matrices.

One obtains the types $B$ and $G$ electrical Serre relations by the standard technique of "folding" the type $A$ electrical Serre relation. These lead to a new species of electrical Lie algebras $\mathfrak{e}_{D}$ defined for any Dynkin diagram $D$. Besides the above results for type $A$, there appear to be other interesting relations between these $\mathfrak{e}_{D}$ and simple Lie algebras. For example, $\mathfrak{e b}_{2}:=\mathfrak{e}_{B_{2}}$ is isomorphic to $\mathfrak{g l}_{2}$. We conjecture (Conjecture 5.2) that, for a finite-type diagram $D$, the $\operatorname{dimension} \operatorname{dim}\left(\mathfrak{e}_{D}\right)$ is always 
equal to the dimension of the maximal nilpotent subalgebra of the semisimple Lie algebra $\mathfrak{g}_{D}$ with Dynkin diagram $D$, and furthermore (Conjecture 5.3) that $\mathfrak{e}_{D}$ is finite-dimensional if and only if $\mathfrak{g}_{D}$ is finite-dimensional. We give the electrical analogue of Lusztig's relation for type $B$ (see [Berenstein and Zelevinsky 1997]) where we observe similar positivity to the totally nonnegative case.

The most interesting case beyond finite Dynkin types is affine type $A$. The corresponding electrical Lie (semi-)group action on planar electrical networks is perhaps even more natural than that of $\mathrm{EL}_{2 n}$, since one can obtain all (rather than just a dense subset of; see Corollary 4.7) response matrices of planar networks in this way [CdVGV 1996; CIM 1998], and furthermore the circular symmetry of the planar networks is preserved. We do not, however, attempt to address this case in the current paper.

\section{Electrical networks}

For more background on electrical networks, we refer the reader to [CIM 1998; CdVGV 1996].

Response matrix. For our purposes, an electrical network is a finite weighted undirected graph $N$, where the vertex set is divided into the boundary vertices and the interior vertices. The weight $w(e)$ of an edge is to be thought of as the conductance of the corresponding resistor, and is generally taken to be a positive real number. A 0 -weighted edge would be the same as having no edge, and an edge with infinite weight would be the same as identifying the endpoint vertices.

We define the Kirchhoff matrix $K(N)$ to be the square matrix with rows and columns labeled by the vertices of $N$, defined by

$$
K_{i j}=\left\{\begin{aligned}
-\sum_{e \text { joins } i \text { and } j} w(e) & \text { for } i \neq j, \\
\sum_{e \text { incident to } i} w(e) & \text { for } i=j .
\end{aligned}\right.
$$

We define the response matrix $L(N)$ to be the square matrix with rows and columns labeled by the boundary vertices of $N$, given by the Schur complement

$$
L(N)=K / K_{I}
$$

where $K_{I}$ denotes the submatrix of $K$ indexed by the interior vertices. The response matrix encodes all the electrical properties of $N$ that can be measured from the boundary vertices. For example, the Kirchhoff matrix and response matrix of the "Y"-graph in Figure 3 (with the surrounding edges removed and the central vertex made interior), are given by

$$
K(N)=\left[\begin{array}{cccc}
a & 0 & 0 & -a \\
0 & b & 0 & -b \\
0 & 0 & c & -c \\
a & b & c & a+b+c
\end{array}\right] \text { and } L(N)=\left[\begin{array}{ccc}
\frac{a(b+c)}{a+b+c} & -\frac{a b}{a+b+c} & -\frac{a c}{a+b+c} \\
-\frac{a b}{a+b+c} & \frac{b(a+c)}{a+b+c} & -\frac{b c}{a+b+c} \\
-\frac{a c}{a+b+c} & -\frac{b c}{a+b+c} & \frac{(a+b) c}{a+b+c}
\end{array}\right] .
$$



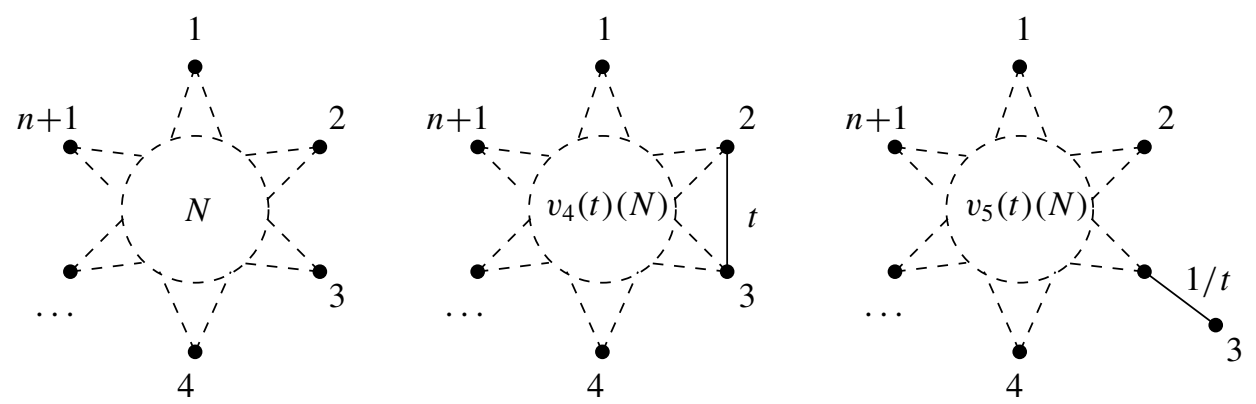

Figure 1. The operations $v_{i}(t)$ acting on a network $N$.

Planar electrical networks. We shall usually consider electrical networks $\mathrm{N}$ embedded in a disk, so that the boundary vertices, numbered $1,2, \ldots, n+1$, lie on the boundary of the disk.

Given an odd integer $2 k-1$, for $k=1,2, \ldots, n+1$, and a nonnegative real number $t$, we define $v_{2 k-1}(t)(N)$ to be the electrical network obtained from $N$ by adding a new edge from a new vertex $v$ to $k$, with weight $1 / t$, followed by treating $k$ as an interior vertex and the new vertex $v$ as a boundary vertex (now named $k$ ).

Given an even integer $2 k$, for $k=1,2, \ldots, n+1$, and a nonnegative real number $t$, we define $v_{2 k}(t)(N)$ to be the electrical network obtained from $N$ by adding a new edge from $k$ to $k+1$ (indices taken modulo $n+1$ ), with weight $t$.

The two operations are shown in Figure 1. In [CIM 1998], these operations are called adjoining a boundary spike, and adjoining a boundary edge respectively. Our notation suggests, and we shall establish, that there is some symmetry between these two types of operations.

In [CIM 1998, §8], it is shown that $L\left(v_{i}(a) \cdot N\right)$ depends only on $L(N)$, giving an operation $v_{i}(t)$ on response matrices. Denote by $x_{i j}$ the entries of the response matrix, where $1 \leq i, j \leq n+1$. In particular, we have $x_{i j}=x_{j i}$. Then, if $\delta_{i j}$ denotes the Kronecker delta, we have

$v_{2 k-1}(t): x_{i j} \mapsto x_{i j}-\frac{t x_{i k} x_{k j}}{t x_{k k}+1}, \quad v_{2 k}(t): x_{i j} \mapsto x_{i j}+\left(\delta_{i k}-\delta_{(i+1) k}\right)\left(\delta_{j k}-\delta_{(j+1) k}\right) t$.

We caution that the parameter $\xi$ in [CIM 1998] is the inverse of our $t$ in the odd case.

Electrically equivalent transformations of networks. Series-parallel transformations are shown in Figure 2. The following proposition is well-known and can be found for example in [CdVGV 1996].
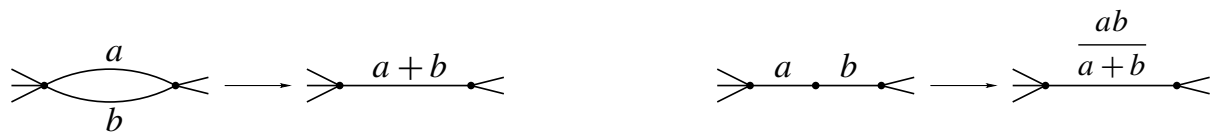

Figure 2. Series-parallel transformations. 

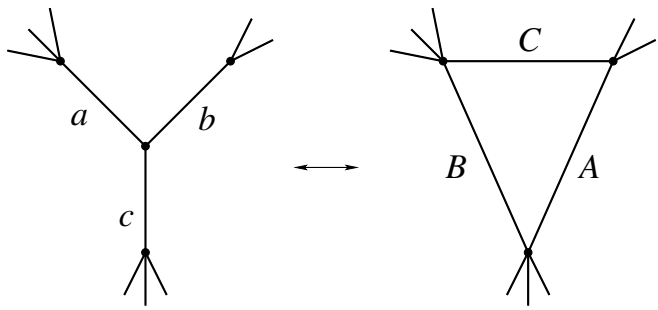

Figure 3. The $Y-\Delta$, or star-triangle, transformation.

Proposition 2.1. Series-parallel transformations, removing loops, and removing interior degree 1 vertices do not change the response matrix of a network.

The following theorem is attributed to Kennelly [1899].

Proposition 2.2 ( $Y-\Delta$ transformation). Assume that the parameters $a, b, c$ and $A, B, C$ are related by

$$
A=\frac{b c}{a+b+c}, \quad B=\frac{a c}{a+b+c}, \quad C=\frac{a b}{a+b+c},
$$

or equivalently by

$$
a=\frac{A B+A C+B C}{A}, \quad b=\frac{A B+A C+B C}{B}, \quad c=\frac{A B+A C+B C}{C} .
$$

Then switching a local part of an electrical network between the two options shown in Figure 3 does not change the response matrix of the whole network.

\section{The electrical Lie algebra $\mathfrak{e l}_{2 n}$}

Let $\mathfrak{e l}_{2 n}$ be the Lie algebra generated over $\mathbb{R}$ by $e_{i}, i=1, \ldots, 2 n$, subject to the relations

- $\left[e_{i}, e_{j}\right]=0$ for $|i-j|>1$,

- $\left[e_{i},\left[e_{i}, e_{j}\right]\right]=-2 e_{i},|i-j|=1$.

Theorem 3.1. The electrical Lie algebra $\mathfrak{e l}_{2 n}$ is isomorphic to the real symplectic Lie algebra $\mathfrak{s p}_{2 n}$.

Proof. We identify the symplectic algebra $\mathfrak{s p}_{2 n}$ with the space of $2 n \times 2 n$ matrices which in block form

$$
\left(\begin{array}{ll}
A & B \\
C & D
\end{array}\right)
$$

satisfy $A=-D^{T}, B=B^{T}$ and $C=C^{T}$ (see [Fulton and Harris 1991, Lecture 16]). Note that the Lie subalgebra consisting of the matrices where $B=C=0$ is naturally isomorphic to $\mathfrak{g l}_{n}$. 
Let $\varepsilon_{i} \in \mathbb{R}^{n}$ denote the standard basis column vector with a 1 in the $i$-th position. Let

$$
a_{1}=\varepsilon_{1}, \quad a_{2}=\varepsilon_{1}+\varepsilon_{2}, \quad a_{3}=\varepsilon_{2}+\varepsilon_{3}, \quad \ldots, \quad a_{n}=\varepsilon_{n-1}+\varepsilon_{n},
$$

and let

$$
b_{1}=\varepsilon_{1}, \quad b_{2}=\varepsilon_{2}, \quad \ldots, \quad b_{n}=\varepsilon_{n} .
$$

For $1 \leq i \leq n$, define elements of $\mathfrak{s p}_{2 n}$ by the formulae

$$
e_{2 i-1}=\left(\begin{array}{cc}
0 & a_{i} \cdot a_{i}^{T} \\
0 & 0
\end{array}\right), \quad e_{2 i}=\left(\begin{array}{cc}
0 & 0 \\
b_{i} \cdot b_{i}^{T} & 0
\end{array}\right) .
$$

We claim that this gives a symplectic representation $\phi$ of $\mathfrak{e l}_{2 n}$ which is an isomorphism of $\mathfrak{e l}_{2 n}$ with $\mathfrak{s p}_{2 n}$. We first check that the relations of $\mathfrak{e l}_{2 n}$ are satisfied. It is clear from the block matrix form that $\left[e_{2 i-1}, e_{2 j-1}\right]=0=\left[e_{2 i}, e_{2 j}\right]$ for any $i, j$. Now,

$$
\left[e_{2 i-1}, e_{2 j}\right]=\left(\begin{array}{cc}
\left(a_{i} \cdot a_{i}^{T}\right)\left(b_{j} \cdot b_{j}^{T}\right) & 0 \\
0 & -\left(b_{j} \cdot b_{j}^{T}\right)\left(a_{i} \cdot a_{i}^{T}\right)
\end{array}\right) .
$$

But, by construction, $b_{j}^{T} \cdot a_{i}=0=a_{i}^{T} \cdot b_{j}$ unless $j=i$ or $j=i-1$. Thus $\left[e_{k}, e_{l}\right]=0$ unless $|k-l|=1$. Finally,

$$
\begin{aligned}
{\left[e_{2 i-1},\left[e_{2 i-1}, e_{2 i}\right]\right] } & =\left(\begin{array}{cc}
0 & -2\left(a_{i} \cdot a_{i}^{T}\right)\left(b_{i} \cdot b_{i}^{T}\right)\left(a_{i} \cdot a_{i}^{T}\right) \\
0 & 0
\end{array}\right) \\
& =\left(\begin{array}{cc}
0 & -2 a_{i} \cdot a_{i}^{T} \\
0 & 0
\end{array}\right)=-2 e_{2 i-1}
\end{aligned}
$$

using the fact that $a_{i}^{T} \cdot b_{i}=1=b_{i}^{T} \cdot a_{i}$. Similarly, one obtains $\left[e_{k},\left[e_{k}, e_{k \pm 1}\right]\right]=-2 e_{k}$. Thus we have a symplectic representation $\phi: \mathfrak{e l}_{2 n} \rightarrow \mathfrak{s p}_{2 n}$.

Now we show that this map is surjective. First we verify that $\mathfrak{g l}_{n} \subset \phi\left(\mathfrak{e l}_{2 n}\right)$. The nonzero commutators $\left[e_{i}, e_{j}\right]$ give matrices of the form $\left(\begin{array}{cc}A & 0 \\ 0 & -A^{T}\end{array}\right)$, where $A$ is a scalar multiple of one of the matrices $E_{1,1}, E_{i, i}+E_{i+1, i}$ or $E_{i+1, i}+E_{i+1, i+1}$. Here $E_{i, j}$ denotes the $n \times n$ matrix with a single nonzero entry equal to 1 in the $(i, j)$-th position. All the matrices of the above form occur. It is easy to see that $\phi\left(\mathfrak{e l}_{2 n}\right)$ must then contain the matrices where $A=E_{i, i}, E_{i, i+1}$ or $E_{i+1, i}$ for each $i$. But these matrices generate $\mathfrak{g l}_{n}$ as a Lie algebra. However, it is known [Humphreys 1990, Proposition 8.4(d)] that for a semisimple Lie algebra $L$, one has $\left[L_{\alpha}, L_{\beta}\right]=L_{\alpha+\beta}$ when $\alpha, \beta, \alpha+\beta$ are all roots and $L_{\alpha}$ denotes a root subspace. It follows easily from the explicit description of the root system of $\mathfrak{s p}_{2 n}$ and the definition of $e_{i}$ that every root subspace of $\mathfrak{s p}_{2 n}$ is contained in $\phi\left(\mathfrak{e l}_{2 n}\right)$, completing the proof.

To see that the map $\phi: \mathfrak{e l}_{2 n} \rightarrow \mathfrak{s p}_{2 n}$ is injective, we note:

Lemma 3.2. The dimension of $\mathfrak{e l}_{2 n}$ is $n(2 n+1)$. 
Proof. According to Lemma 5.1, the dimension of $\mathfrak{e l}_{2 n}$ is at most $n(2 n+1)$. On the other hand, we just saw that the map $\mathfrak{e l}_{2 n} \rightarrow \mathfrak{s p}_{2 n}$ is surjective. The statement follows.

\section{The electrical Lie group $\mathrm{EL}_{2 n}$}

Let $\mathrm{EL}_{2 n}$ be a split real Lie group with Lie algebra $\mathfrak{e l}_{2 n}$. For concreteness, we shall choose $\mathrm{EL}_{2 n}$ to be the real symplectic group, but we will use the notation $\mathrm{EL}_{2 n}$ to remind the reader that the generators we consider are associated to the presentation of $\mathfrak{e l}_{2 n}$, rather than the usual presentation of $\mathfrak{s p}_{2 n}$. Let $u_{i}(a)=\exp \left(a e_{i}\right)$ denote the one-parameter subgroups corresponding to the generators of $\mathfrak{e l}_{2 n}$.

Theorem 4.1. The elements $u_{i}(a)$ satisfy the relations

(1) $u_{i}(a) u_{i}(b)=u_{i}(a+b)$,

(2) $u_{i}(a) u_{j}(b)=u_{j}(b) u_{i}(a)$ if $|i-j|>1$, and

(3) $u_{i}(a) u_{j}(b) u_{i}(c)=u_{j}(b c /(a+c+a b c)) u_{i}(a+c+a b c) u_{j}(a b /(a+c+a b c))$ if $|i-j|=1$.

Proof. The first two relations are clear. For the third, observe that, for each $1 \leq i \leq 2 i-1$, the elements $e_{i}, e_{i+1}$, and $\left[e_{i}, e_{i+1}\right]$ are the usual Chevalley generators of a Lie subalgebra isomorphic to $\mathfrak{s l}_{2}$. Thus we can verify the relation inside $\mathrm{SL}_{2}(\mathbb{R})$ :

$$
\begin{aligned}
\left(\begin{array}{ll}
1 & a \\
0 & 1
\end{array}\right)\left(\begin{array}{ll}
1 & 0 \\
b & 1
\end{array}\right)\left(\begin{array}{ll}
1 & c \\
0 & 1
\end{array}\right) & =\left(\begin{array}{cc}
1+a b & a+c+a b c \\
b & 1+b c
\end{array}\right) \\
& =\left(\begin{array}{cc}
1 & 0 \\
\frac{b c}{a+c+a b c} & 1
\end{array}\right)\left(\begin{array}{cc}
1 & a+c+a b c \\
0 & 1
\end{array}\right)\left(\begin{array}{cc}
1 & 0 \\
\frac{a b}{a+c+a b c} & 1
\end{array}\right) .
\end{aligned}
$$

Remark. There is a one-parameter deformation which connects the relation in Theorem 4.1(3) with Lusztig's relation [1994] in total positivity:

$$
u_{i}(a) u_{j}(b) u_{i}(c)=u_{j}\left(\frac{b c}{a+c}\right) u_{i}(a+c) u_{j}\left(\frac{a b}{a+c}\right) .
$$

Consider the associative algebra $U_{\tau}\left(\mathfrak{e l}_{2 n}\right)$ where the generators $\varepsilon_{1}, \varepsilon_{2}, \ldots, \varepsilon_{2 n}$ satisfy the following deformed Serre relations:

- $\varepsilon_{i} \varepsilon_{j}=\varepsilon_{j} \varepsilon_{i}$ if $|i-j|>1$,

- $\varepsilon_{i} \varepsilon_{j} \varepsilon_{i}=\tau \varepsilon_{i}+\frac{1}{2}\left(\varepsilon_{i}^{2} \varepsilon_{j}+\varepsilon_{j} \varepsilon_{i}^{2}\right)$ if $|i-j|=1$.

This is a one-parameter family of algebras which at $\tau=0$ reduces to $U\left(\mathfrak{n}^{+}\right)$, where $\mathfrak{s l}_{2 n+1}=\mathfrak{n}^{-} \oplus \mathfrak{h} \oplus \mathfrak{n}^{+}$is the Cartan decomposition, while at $\tau=1$ it gives the universal enveloping algebra $U\left(\mathfrak{e l}_{2 n}\right)$ of the electrical Lie algebra. For $U_{\tau}\left(\mathfrak{e l}_{2 n}\right)$, the "braid move" for the elements $\exp \left(a \varepsilon_{i}\right)$ then takes the form

$$
u_{i}(a) u_{j}(b) u_{i}(c)=u_{j}\left(\frac{b c}{a+c+\tau a b c}\right) u_{i}(a+c+\tau a b c) u_{j}\left(\frac{a b}{a+c+\tau a b c}\right)
$$


if $|i-j|=1$. At $\tau=0$ this reduces to (2), and at $\tau=1$ it is the relation Theorem 4.1(3).

Nonnegative part of $\mathbf{E L}_{2 n}$. Define the nonnegative part $\left(\mathrm{EL}_{2 n}\right)_{\geq 0}$ of $\mathrm{EL}_{2 n}$, or equivalently the electrically nonnegative part of $\mathrm{Sp}_{2 n}$, to be the subsemigroup generated by the $u_{i}(a)$ with nonnegative parameters $a$.

For a reduced word $\boldsymbol{i}=i_{1} \cdots i_{\ell}$ of $w \in S_{2 n+1}$, denote by $E(w) \subset\left(\mathrm{EL}_{2 n}\right)_{\geq 0}$ the image of the map

$$
\psi_{i}:\left(a_{1}, \ldots, a_{\ell}\right) \in \mathbb{R}_{>0}^{\ell} \mapsto u_{i_{1}}\left(a_{1}\right) \ldots u_{i_{\ell}}\left(a_{\ell}\right) .
$$

It follows from the relations in Theorem 4.1 that the set $E(w)$ depends only on $w$, and not on the chosen reduced word. The following proposition is similar to [Lusztig 1994, Proposition 2.7], which gives a decomposition $U_{\geq 0}=\bigsqcup_{w} U_{\geq 0}^{w}$ of the totally nonnegative part of the unipotent group.

Proposition 4.2. The sets $E(w)$ are disjoint and cover the whole $\left(\mathrm{EL}_{2 n}\right)_{\geq 0}$. Each of the maps $\psi_{i}: \mathbb{R}_{>0}^{\ell} \rightarrow E(w)$ is a homeomorphism.

Proof. Using Theorem 4.1, we can rewrite any product of generators $u_{i}(a)$ by performing braid moves similar to those in the symmetric group $S_{2 n+1}$. Any product of the generators can be transformed into a product that corresponds to a reduced word in $S_{2 n+1}$, and thus it belongs to one of the $E(w)$.

If the map $\psi_{i}$ is not injective for some reduced word $\boldsymbol{i}$, then we can find two reduced products

$$
u_{i_{1}}\left(a_{1}\right) \cdots u_{i_{\ell}}\left(a_{\ell}\right)=u_{i_{1}}\left(b_{1}\right) \cdots u_{i_{\ell}}\left(b_{\ell}\right)
$$

for two $\ell$-tuples of positive numbers such that $a_{1} \neq b_{1}$. Without loss of generality we can assume $a_{1}>b_{1}$, and thus

$$
u_{i_{1}}\left(a_{1}-b_{1}\right) u_{i_{2}}\left(a_{2}\right) \cdots u_{i_{\ell}}\left(a_{\ell}\right)=u_{i_{2}}\left(b_{2}\right) \cdots u_{i_{\ell}}\left(b_{\ell}\right) .
$$

This shows that two different $E(w)$ 's have nonempty intersection. Thus it suffices to show that the latter is impossible.

Furthermore, it suffices to prove that the top cell corresponding to the longest element $w_{0}$ does not intersect any other cell. Indeed, if any two cells intersect, by adding some extra factors to both we can lift it to the top cell intersecting one of the other cells. Assume we have a product of the form

$$
u=\left[u_{1}\left(a_{1}\right)\right]\left[u_{2}\left(a_{2}\right) u_{1}\left(a_{3}\right)\right] \cdots\left[u_{k}\left(a_{\ell-k+1}\right) \cdots u_{1}\left(a_{\ell}\right)\right],
$$

where $\ell=\left(\begin{array}{l}k \\ 2\end{array}\right)$. Let $\Phi(u) \in \mathrm{Sp}_{2 n}$ be the image of $u$ in $\mathrm{Sp}_{2 n}$ under the natural map $\mathrm{EL}_{2 n} \rightarrow \mathrm{Sp}_{2 n}$ induced by the map $\phi: \mathfrak{e l}_{2 n} \rightarrow \mathfrak{s p}_{2 n}$ of Theorem 3.1. We argue that the positive parameters $a_{\ell-k+1}, \ldots, a_{\ell}$ can be recovered uniquely from just 
looking at the $(n+k / 2)$-th row of $\Phi(u)$ for $k$ even, or the $(k+1) / 2$-th row for $k$ odd. Furthermore, the same calculation will tell us if exactly one of them is equal to zero.

Indeed, assume $k$ is odd. Then the $n+(k+1) / 2$-th entry in the $(k+1) / 2$-th row is just equal to $a_{\ell-k+1}$. Next, once we know $a_{\ell-k+1}$, we can use $(k-1) / 2$-th entry of the same row to recover $a_{\ell-k+2}$, after which we can use $n+(k-1) / 2$-th entry to recover $a_{\ell-k+3}$, and so on. For each step we solve a linear equation where the parameter we divide by is strictly positive as long as all previous parameters $a_{i}$ recovered are positive. For example, let $n=2$ and take the product

$$
u_{1}\left(a_{1}\right) u_{2}\left(a_{2}\right) u_{1}\left(a_{3}\right) u_{3}\left(a_{4}\right) u_{2}\left(a_{5}\right) u_{1}\left(a_{6}\right) .
$$

Then the second row of this product is $\left(a_{4} a_{5}, 1, a_{4}+a_{4} a_{5} a_{6}, a_{4}\right)$. The last entry tells us $a_{4}$, then from the first entry we solve for $a_{5}$, then from the third entry we solve for $a_{6}$. The case of even $k$ is similar; the order in which we have to read the entries of the $(n+k / 2)$-th row in this case is $k / 2-$ th, $(n+k / 2)$-th, $(k / 2-1)$-th, $(n+k / 2-1)$-th, etc. For example, in the product

$$
u_{1}\left(a_{1}\right) u_{2}\left(a_{2}\right) u_{1}\left(a_{3}\right) u_{3}\left(a_{4}\right) u_{2}\left(a_{5}\right) u_{1}\left(a_{6}\right) u_{4}\left(a_{7}\right) u_{3}\left(a_{8}\right) u_{2}\left(a_{9}\right) u_{1}\left(a_{10}\right),
$$

the last row is $\left(a_{7} a_{8} a_{9}, a_{7}, a_{7} a_{8}+a_{7} a_{8} a_{9} a_{10}, 1+a_{7} a_{8}\right)$. By looking at second, last, first and third entries we can solve for $a_{7}, a_{8}, a_{9}$ and $a_{10}$ one after the other.

Now we are ready to complete the argument. Assume that $E\left(w_{0}\right)$ intersects some other $E(v)$. For any reduced word of $w_{0}$, one can find a subword which is a reduced word for $v$, so that we have

$$
\begin{aligned}
u & =\left[u_{1}\left(a_{1}\right)\right]\left[u_{2}\left(a_{2}\right) u_{1}\left(a_{3}\right)\right] \cdots\left[u_{k}\left(a_{\ell-k+1}\right) \cdots u_{1}\left(a_{\ell}\right)\right] \\
& =\left[u_{1}\left(b_{1}\right)\right]\left[u_{2}\left(b_{2}\right) u_{1}\left(b_{3}\right)\right] \cdots\left[u_{k}\left(b_{\ell-k+1}\right) \cdots u_{1}\left(b_{\ell}\right)\right],
\end{aligned}
$$

where the $a_{i}$ are all positive, but the $b_{i}$ are nonnegative, with at least one zero. The above algorithm of recovering the $a_{i}$ will arrive at a contradiction. Thus $E\left(w_{0}\right)$ is disjoint from all other $E(v)$.

It remains to show that $\phi_{i}^{-1}$ is continuous for any reduced word. If $\boldsymbol{i}$ is the reduced word of $w_{0}$ used in (3), this follows from the algorithm above: the $a_{i}$ depend continuously on the matrix entries of $\Phi(u)$. But any two reduced words are connected by braid and commutation moves, so it follows from the (continuously invertible) formulae in Theorem 4.1 that $\phi_{i}^{-1}$ is continuous for any reduced word of $w_{0}$. But for any other $v \in S_{2 n+1}$, a reduced word $j$ for $v$ can be found as an initial subword of some reduced word $i$ for $w_{0}$. The map $\phi_{j}^{-1}$ can then be expressed as a composition of right multiplication by a fixed element of $\mathrm{EL}_{2 n}$, the map $\phi_{i}^{-1}$ and the projection from $\mathbb{R}_{>0}^{\ell\left(w_{0}\right)}$ to $\mathbb{R}_{>0}^{\ell(v)}$, all of which are continuous.

Action on electrical networks. Let $\mathscr{P}(n+1) \subset \mathbb{R}^{(n+1)^{2}}$ denote the set of response matrices of planar electrical networks with $n+1$ boundary vertices. In [CIM 1998], 

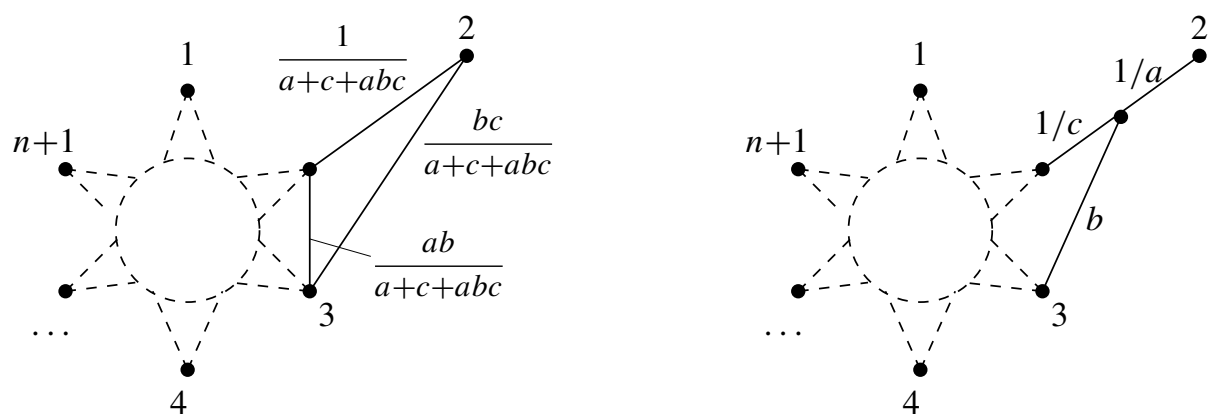

Figure 4. Action of two products on an electrical network.

it is shown that $\mathscr{P}(n+1)$ is exactly the set of symmetric, circular totally nonnegative $(n+1) \times(n+1)$ matrices with row sums equal to 0 . A matrix $M$ is circular totally nonnegative if the signed minors $(-1)^{k} A_{\left(p_{1}, p_{2}, \ldots, p_{k}\right),\left(q_{1}, q_{2}, \ldots, q_{k}\right)}$ are all nonnegative whenever $\left(p_{1}, p_{2}, \ldots, p_{k}, q_{k}, q_{k-1}, \ldots, q_{1}\right)$ is in circular order. In [CdVGV 1996, Théorème 4], it is shown that $\mathscr{P}(n+1)$ can be identified with the set of planar electrical networks with $n+1$ boundary vertices modulo the local transformations described at the end of Section 2. Let $N_{0}$ denote the empty network (with $n+1$ boundary vertices) and let $L_{0}=L\left(N_{0}\right)$ denote the zero matrix.

Theorem 4.3. The nonnegative part $\left(\mathrm{EL}_{2 n}\right)_{\geq 0}$ of the electrical group acts on $\mathscr{P}(n+1)$ via

$$
u_{i}(a) \cdot L(N)=L\left(v_{i}(a)(N)\right) .
$$

Proof. For a single generator $u_{i}(a)$, the stated action is well-defined because it can be described explicitly on the level of response matrices. The formula for $L\left(v_{i}(a)(N)\right)$ in terms of $L(N)$ is given by (1).

We first show that the relations of Theorem 4.1 hold for this action. Relation (1) follows from the series-parallel relation for networks. Relation (2) is immediate: the corresponding networks are identical without any transformations. Relation (3) follows from the $Y-\Delta$ transformation (see Example 4.4).

But now suppose we have two different expressions for $u \in\left(\mathrm{EL}_{2 n}\right)_{\geq 0}$ in terms of generators. Then using relations (1)-(3) of Theorem 4.1, we may assume that both expressions are products corresponding to a reduced word. By Proposition 4.2, the two products come from reduced words for the same $w \in S_{2 n+1}$. It follows that they are related by relations (1)-(3).

Example 4.4. The products

$$
u_{3}(a) u_{4}(b) u_{3}(c) \text { and } u_{4}\left(\frac{b c}{a+c+a b c}\right) u_{3}(a+c+a b c) u_{4}\left(\frac{a b}{a+c+a b c}\right)
$$

act on a network in exactly the same way, as shown in Figure 4. 
A permutation $w=w(1) w(2) \cdots w(2 n+1) \in S_{2 n+1}$ is efficient if

(1) $w(1)<w(3)<\cdots<w(2 n+1)$ and $w(2)<w(4)<\cdots<w(2 n)$, and

(2) $w(1)<w(2), w(3)<w(4), \ldots, w(2 n-1)<w(2 n)$.

Recall the left weak order of permutations is given by $w \preceq v$ if and only if there is a $u$ so that $u w=v$ and $\ell(v)=\ell(u)+\ell(w)$. It is a standard fact that $w \preceq v$ if and only if $v(i)>v(j)$ whenever $w(i)>w(j)$ and $i<j$. Thus the set of efficient permutations has a maximum in left weak order, namely $w=1(n+2) 2(n+3) \cdots(n)(2 n+1)(n+1)$ with length $\frac{1}{2} n(n-1)$.

Theorem 4.5. Let $w \in S_{2 n+1}$. The map $\Theta_{w}: E(w) \rightarrow \mathscr{P}(n+1)$ given by $\Theta_{w}(u)=$ $u \cdot L_{0}$ is injective if and only if $w$ is efficient. We have image $\left(\Theta_{w}\right) \cap$ image $\left(\Theta_{v}\right)=\varnothing$ for $w \neq v$ both efficient. If $w$ is not efficient, there is a unique efficient $v$ such that $\operatorname{image}\left(\Theta_{w}\right)=\operatorname{image}\left(\Theta_{v}\right)$.

Proof. Let $w^{*} \in S_{2 n+1}$ be the efficient permutation of maximal length. Then a possible reduced word for $w^{*}$ is

$$
(n+1) \cdots(46 \ldots(2 n-2))(35 \ldots(2 n-1))(246 \ldots(2 n)) .
$$

The graph obtained by taking the corresponding $u_{i}(a)$ and acting on the empty network is exactly the "standard graph" of [CIM 1998, §7] or the graph $C_{N}$ of [CdVGV 1996]. In particular, $\Theta_{w^{*}}$ is injective by [CIM 1998, Theorem 2] or [CdVGV 1996, Théorème 3]. Suppose $w$ is an arbitrary efficient permutation. Then since $w^{*}=u w$ for some $u \in S_{2 n+1}$, if $\Theta_{w}$ is not injective then $\Theta_{w^{*}}$ is not injective as well, so we conclude that $\Theta_{w}$ is injective.

For a pair $(i, j)$ with $1 \leq i<j \leq n+1$, let us say that a network $N$ is $(i, j)$ connected if we can find a disjoint set of paths $p_{1}, p_{2}, \ldots, p_{\lfloor(j-i+1) / 2\rfloor}$ so that for each $k$, the path $p_{k}$ connects boundary vertex $i+k-1$ to boundary vertex $j-k+1$ without passing through any other boundary vertex. This is a special case of the connections of circular pairs $(P, Q)$ of [CIM 1998]. Let $N_{w}$ be a graph constructed from some reduced word of an efficient $w$. We observe that $N_{w}$ is $(i, j)$-connected if and only if $w(2 i)>w(2 j-1)$.

For example, the first network in Figure 5 corresponds to $w=s_{5} s_{3} s_{6} s_{4} s_{2}=$ $(1,3,5,2,7,4,6)$. We see that it is $(2,4)$-connected, which agrees with the inequality $w(4)=6>5=w(7)$. If the dashed edge is not there, we have $w=s_{3} s_{6} s_{4} s_{2}=(1,3,5,2,4,7,6)$ and $w(4)=5<6=w(7)$, in agreement with the network not being $(2,4)$-connected. Similarly, the second network in Figure 5 corresponds to $w=s_{4} s_{5} s_{3} s_{6} s_{4} s_{2}=(1,3,5,7,2,4,6)$ with the dashed edge and to $w=s_{5} s_{3} s_{6} s_{4} s_{2}=(1,3,5,2,7,4,6)$ without. In the first case it is $(1,4)$-connected, in the second it is not, in agreement with relative order of $w(2)$ and $w(7)$.

Note that the inversions $w(2 i)>w(2 j-1)$ are exactly the possible inversions of an efficient permutation. Since $w$ is determined by its inversions, it follows that the 



Figure 5. Networks and connections on them.

set of $(i, j)$-connections of $N_{w}$ determines $w$, and that image $\left(\Theta_{w}\right) \cap$ image $\left(\Theta_{v}\right)=\varnothing$ for $w \neq v$ both efficient.

Suppose $w$ is not efficient. Then it is easy to see that $w$ has a reduced expression $s_{i_{1}} s_{i_{2}} \cdots s_{i_{\ell}}$, where either (1) $i_{\ell}$ is odd, or (2) $i_{\ell}$ is even and $i_{\ell}=i_{\ell-1} \pm 1$. But $u_{i}(a) \cdot L_{0}=L_{0}$ for odd $i$ since all the boundary vertices are still disconnected in $u_{i}(a) \cdot N_{0}$, and for even $i$ we have $u_{i \pm 1}(a) u_{i}(b) \cdot L_{0}=u_{i}(1 / a+b) L_{0}$, using the series-transformation (Proposition 2.1). It follows that $\Theta_{w}$ is not injective. Furthermore, image $\left(\Theta_{w}\right)=\operatorname{image}\left(\Theta_{v}\right)$, where $v$ is obtained from $w$ by recursively (1) removing $i_{\ell}$ from a reduced word of $w$ if $i_{\ell}$ is odd, or (2) changing the last two letters $\left(i_{\ell} \pm 1\right) i_{\ell}$ to $i_{\ell}$ when $i_{\ell}$ is even. An efficient $v$ obtained in this way must be unique, since image $\left(\Theta_{v}\right) \cap$ image $\left(\Theta_{v^{\prime}}\right)=\varnothing$ for efficient $v \neq v^{\prime}$.

Corollary 4.6. The number of efficient $w \in S_{2 n+1}$ is equal to the Catalan number $C_{n+1}=\frac{1}{n+2}\left(\begin{array}{c}2 n+2 \\ n+1\end{array}\right)$.

Proof. It is clear that $(2 i, 2 j+1)$ is an inversion of $w$ only if $(2 i, 2 j-1)$ and $(2 i+2,2 j+1)$ are also inversions, and this characterizes inversion sets of efficient $w$. Thus the set of efficient $w \in S_{2 n+1}$ is in bijection with the lower order ideals of the positive root poset of $\mathfrak{s l}_{n+1}$, which is well known to be enumerated by the Catalan number [Fomin and Reading 2007].

Corollary 4.7. $\left(\mathrm{EL}_{2 n}\right)_{\geq 0} \cdot L_{0}$ is dense in $\mathscr{P}(n+1)$.

Proof. Follows from Theorem 4.5 and [CdVGV 1996, Théorème 5].

\section{Stabilizer.}

Lemma 4.8. The stabilizer subsemigroup of $\left(\mathrm{EL}_{2 n}\right)_{\geq 0}$ acting on the zero matrix $L_{0}$ is the subsemigroup generated by $u_{2 i+1}(a)$ for all $a \in \mathbb{R}_{\geq 0}$ and all $i$.

Proof. It is clear that $u_{2 i+1}(a)$ lies in the stabilizer. But the action of any $u_{2 i}(a)$ will change the connectivity of the network, and it is impossible to return to trivial connectivity by adding more edges, or by relations. 
The semigroup stabilizer is too small in the sense that it does not detect the relations $u_{i \pm 1}(a) u_{i}(b) \cdot L_{0}=u_{i}(1 / a+b) L_{0}$ used in the proof of Theorem 4.5 . We shall calculate the stabilizer subalgebra of the corresponding infinitesimal action of the Lie algebra $\mathfrak{e l}_{2 n}$, which will in particular give an algebraic explanation of these relations. The reason we do not work with the whole Lie group $\mathrm{EL}_{2 n}$ is threefold: (1) nonpositive elements of $\mathrm{EL}_{2 n}$ will produce networks that are "virtual", that is, that have negative edge weights; (2) the topology of $\mathrm{EL}_{2 n}$ means that to obtain an action one cannot just check the relations of Theorem 4.1; (3) when the parameters are nonpositive, the relation Theorem 4.1(3) develops singularities.

To describe the infinitesimal action of $\mathfrak{e l}_{2 n}$, we give derivations of $\mathbb{R}\left[x_{i j}\right]$, the polynomial ring in $\frac{1}{2}(n+1)(n+2)$ variables $x_{i j}$ where $1 \leq i, j \leq n+1$ and we set $x_{i j}=x_{j i}$.

Proposition 4.9. The electrical Lie algebra $\mathfrak{e l}_{2 n}$ acts on $\mathbb{R}\left[x_{i j}\right]$ via the derivations

$$
\begin{aligned}
e_{2 i} & \mapsto \partial_{i i}+\partial_{i+1, i+1}-\partial_{i, i+1}, \\
e_{2 i-1} & \mapsto-\sum_{1 \leq p \leq q \leq n+1} x_{i p} x_{i q} \cdot \partial_{p q}
\end{aligned}
$$

Proof. These formulae can be checked by directly verifying the defining relations of $\mathfrak{e l}_{2 n}$. Alternatively, they can be deduced by differentiating the formulae (1).

We calculate that

$$
\left[e_{2 i}, e_{2 i-1}\right] \mapsto-x_{i i} \partial_{i i}+x_{i, i+1} \partial_{i+1, i+1}+\sum_{1 \leq p \leq n+1}\left(x_{i+1, p} \partial_{i+1, p}-x_{i p} \partial_{i p}\right) .
$$

Theorem 4.10. The stabilizer subalgebra $\mathfrak{e l}_{2 n}^{0}$, at the zero matrix $L_{0}$, of the infinitesimal action of $\mathfrak{e l}_{2 n}$ on the space of response matrices is generated by $e_{i}$ for $i$ odd, and $\left[e_{2 i-1}, e_{2 i}\right]$ for $i=1,2, \ldots, n$.

Proof. The fact the stated elements lie in $\mathfrak{e l}_{2 n}^{0}$ follows from (5) and (6), since $x_{i j}=0$ at the zero matrix. By Lemma 3.2, the elements $e_{\alpha}$ in the proof of Lemma 5.1 form a basis of $\mathfrak{e l}_{2 n}$. Write $\alpha_{i j}:=\alpha_{i}+\alpha_{i+1}+\cdots+\alpha_{j}$ for $1 \leq i \leq j \leq 2 n$ to denote the positive roots of $\mathfrak{s l}_{2 n+1}$. Then we know that $e_{\alpha_{i, i}} \in \mathfrak{e l}_{2 n}^{0}$ for $i$ odd, and $e_{\alpha_{i, i+1}} \in \mathfrak{e l}_{2 n}^{0}$ for each $i$. It follows easily that $e_{\alpha_{i, j}} \in \mathfrak{e l}_{2 n}^{0}$ for every pair $1 \leq i \leq j \leq 2 n$ where at least one of $i$ and $j$ are odd. It follows that

$\operatorname{dim}_{\mathbb{R}}\left(\mathfrak{e l}_{2 n}\right)-\operatorname{dim}_{\mathbb{R}}\left(\mathfrak{e l}_{2 n}^{0}\right) \leq \#\{(i, j) \mid 1 \leq i \leq j \leq 2 n$ and $i, j$ are even $\}=\frac{1}{2} n(n+1)$.

By Theorem 4.5, the action of $\left(\mathrm{EL}_{2 n}\right)_{\geq 0}$ on the zero matrix $L_{0}$ gives a space of dimension $\frac{1}{2} n(n+1)$. It follows that the above inequality is an equality, and that $\mathfrak{e} \mathfrak{l}_{2 n}^{0}$ is generated by the stated elements.

Note that a basis for $\mathfrak{e l}_{2 n} / \mathfrak{e l}_{2 n}^{0}$ is given by the $e_{\alpha_{i, j}}$ where $i$ and $j$ are both even. 
These $\alpha_{i, j}$ are exactly the inversions of efficient permutations (see the proof of Theorem 4.5).

\section{Electrical Lie algebras of finite type}

Dimension. Let $D$ be a Dynkin diagram of finite type and let $A=\left(a_{i j}\right)$ be the associated Cartan matrix. To each node $i$ of $D$ associate a generator $e_{i}$, and define $\mathfrak{e}_{D}$ to be the Lie algebra generated over $\mathbb{R}$ by the $e_{i}$, subject to the relations, for each $i \neq j$,

$$
\begin{cases}\operatorname{ad}\left(e_{i}\right)^{1-a_{i j}}\left(e_{j}\right)=0 & \text { if } a_{i j} \neq-1 \\ \operatorname{ad}\left(e_{i}\right)^{2}\left(e_{j}\right)=-2 e_{i} & \text { if } a_{i j}=-1\end{cases}
$$

These "electrical Serre relations" can be deduced from the type $A$ electrical Serre relations of Section 3 by folding. Namely, the relation for an edge of multiplicity two $\left(a_{i j}=-2\right)$ can be obtained by finding the relation for the elements $e_{2}$ and $e_{1}+e_{3}$ inside $\mathfrak{e l}_{3}$. Similarly, the $\mathfrak{e}_{G_{2}}$ relation $\left(a_{i j}=-3\right)$ can be obtained by finding the relation for the elements $e_{1}+e_{2}+e_{3}$ and $e_{4}$ inside $\mathfrak{e}_{D_{4}}$, where $e_{4}$ corresponds to the node of valency three in $D_{4}$.

Let $\mathfrak{u}_{D}$ denote the nilpotent Lie subalgebra of the simple Lie algebra of type $D$. It is well known that $\mathfrak{u}_{D}$ is generated by Chevalley generators $\tilde{e}_{i}$ subject to the Serre relations $\operatorname{ad}\left(\tilde{e}_{i}\right)^{1-a_{i j}}\left(\tilde{e}_{j}\right)=0$ for each $i \neq j$. Thus the electrical Lie algebra $\mathfrak{e}_{D}$ is a deformation of $\mathfrak{u}_{D}$, and it follows from general results that $\operatorname{dim} \mathfrak{e}_{D} \leq \operatorname{dim} \mathfrak{u}_{D}$. For future reference, we include a direct elementary proof of this fact.

Lemma 5.1. The dimension of the Lie algebra $\mathfrak{e}_{D}$ does not exceed the number of positive roots in the finite root system associated to $D$.

Proof. The Lie algebra $\mathfrak{u}_{D}$ has a basis $\tilde{e}_{\alpha}$ labeled by positive roots $\alpha \in R^{+}$of the root system, where the $\tilde{e}_{i}$ correspond to simple roots. Let us fix an expression

$$
\tilde{e}_{\alpha}=\left[\tilde{e}_{i_{1}},\left[\tilde{e}_{i_{2}},\left[\ldots,\left[\tilde{e}_{i_{l-1}}, \tilde{e}_{i_{l}}\right] \ldots\right]\right]\right]
$$

of shortest possible length for each $\tilde{e}_{\alpha}$, and define the corresponding

$$
e_{\alpha}=\left[e_{i_{1}},\left[e_{i_{2}},\left[\ldots,\left[e_{i_{l-1}}, e_{i_{l}}\right] \ldots\right]\right]\right]
$$

in $\mathfrak{e}_{D}$. We claim that the $e_{\alpha} \operatorname{span} \mathfrak{e}_{D}$. Indeed, it is enough to show that any expression $\left[e_{j_{1}},\left[e_{j_{2}},\left[\ldots,\left[e_{j_{\ell-1}}, e_{j_{\ell}}\right] \ldots\right]\right]\right]$ lies in the linear span of the $e_{\alpha}$. Assume otherwise, and take a counterexample of smallest possible total length $\ell$. Let us define $\hat{e}_{\alpha}$ in the free Lie algebra $\mathfrak{f}_{D}$ with generators $\hat{e}_{i}$ using (7). Then in $\mathfrak{f}_{D}$ we have a relation of the form

$$
\left[\hat{e}_{j_{1}},\left[\hat{e}_{j_{2}},\left[\ldots,\left[\hat{e}_{j_{\ell-1}}, \hat{e}_{j_{\ell}}\right] \ldots\right]\right]\right]-\sum_{\alpha} c_{\alpha} \hat{e}_{\alpha}=\hat{x} \in I
$$


where $I$ denotes the ideal generated by the Serre relations, so that $\mathfrak{u}_{D}=\mathfrak{f}_{D} / I$. Now $\mathfrak{f}_{D}$ is naturally $\mathbb{Z}$-graded, and $I$ is a graded ideal, so we may assume all terms in the relation are homogeneous with the same degree. Now replacing each instance of the Serre relation in $x$ with the corresponding electrical Serre relation gives us a relation

$$
\left[e_{j_{1}},\left[e_{j_{2}},\left[\ldots,\left[e_{j_{\ell-1}}, e_{j_{\ell}}\right] \ldots\right]\right]\right]-\sum_{\alpha} c_{\alpha} e_{\alpha}=x
$$

in $\mathfrak{e}_{D}$, where $x$ is a sum of terms of the form $\left[e_{k_{1}},\left[e_{k_{2}},\left[\ldots,\left[e_{k_{\ell-1}}, e_{k_{\ell^{\prime}}}\right] \ldots\right]\right]\right]$ with $\ell^{\prime}<\ell$, and thus by assumption lies in the span of the $e_{\alpha}$. The statement of the lemma follows.

Conjecture 5.2. The dimension of $\mathfrak{e}_{D}$ coincides with the number of positive roots in the root system of $D$.

A proof of Conjecture 5.2 for Dynkin diagrams of classical type has been announced by Yi Su. One can also define the Lie algebras $\mathfrak{e}_{D}$ for any Dynkin diagram $D$, of finite type or not.

Conjecture 5.3. The Lie algebra $\mathfrak{e}_{D}$ is finite-dimensional if and only if $D$ is of finite type.

Examples. We illustrate Conjecture 5.2 with some examples. For electrical type $A_{2 n}$ it has already been verified in Theorem 3.1.

Electrical $B_{2}$. Consider the case when $D$ has two nodes connected by a double edge. In that case we denote by $\mathfrak{e}_{D}=\mathfrak{e b}_{2}$ the Lie algebra generated by two generators $e$ and $f$ subject to the relations

$$
[e,[e,[e, f]]]=0, \quad[f,[f, e]]=-2 f .
$$

Lemma 5.4. The Lie algebras $\mathfrak{e b}_{2}$ and $\mathfrak{g l}_{2}$ are isomorphic.

Proof. Consider the map

$$
e \mapsto\left(\begin{array}{ll}
1 & 1 \\
0 & 1
\end{array}\right), \quad f \mapsto\left(\begin{array}{ll}
0 & 0 \\
1 & 0
\end{array}\right)
$$

One easily checks that it is a Lie algebra homomorphism, and that it is surjective. By Lemma 5.1 we know the dimension of $\mathfrak{e b}_{2}$ is at most four, so the map must be an isomorphism.

Electrical $G_{2}$. Consider the Lie algebra $\mathfrak{e g}_{2}$ generated by two generators $e$ and $f$ subject to the relations

$$
[e,[e,[e,[e, f]]]]=0, \quad[f,[f, e]]=-2 f .
$$

Lemma 5.5. The Lie algebra $\mathfrak{e g}_{2}$ is six-dimensional. 
Proof. According to Lemma 5.1 the elements

$$
e, f,[e f],[e[e f]],[e[e[e f]]],[f[e[e[e f]]]]
$$

form a spanning set for $\mathfrak{e g}_{2}$. Thus it remains to check that they are linearly independent. This is easily done inside the following faithful representation of $\mathfrak{e g}_{2}$ in $\mathfrak{g l}_{4}$ :

$$
e \mapsto\left(\begin{array}{cccc}
1 & 1 & 0 & 1 \\
0 & 1 & 1 & 0 \\
0 & 0 & 1 & 0 \\
0 & 0 & 0 & 1
\end{array}\right), \quad f \mapsto\left(\begin{array}{llll}
0 & 0 & 0 & 0 \\
0 & 0 & 0 & 0 \\
0 & 0 & 0 & 0 \\
1 & 0 & 0 & 0
\end{array}\right)
$$

Electrical $C_{3}$. Consider the Lie algebra $\mathfrak{e c}_{3}$ generated by three generators $e, f$ and $g$ subject to the relations

$$
\begin{gathered}
{[e,[e,[e, f]]]=0, \quad[f,[f, e]]=-2 f,} \\
{[f,[f, g]]=-2 f, \quad[g,[g, f]]=-2 g, \quad[e, g]=0 .}
\end{gathered}
$$

Lemma 5.6. The Lie algebra $\mathfrak{e c}_{3}$ is nine-dimensional.

Proof. We consider two representations of $\mathfrak{e c}_{3}$ : one inside $\mathfrak{s l}_{9}$ and one inside $\mathfrak{s l}_{2}$. Let $E_{i j}$ denote a matrix with a 1 in the $(i, j)$-th position and 0 s elsewhere. For the first representation, we define

$$
\begin{aligned}
& e \mapsto E_{42}+E_{54}+E_{76}+E_{87}+E_{89}, \\
& f \mapsto 2 E_{24}-2 E_{26}+2 E_{41}+2 E_{45}-E_{47}-2 E_{63}+E_{67}+E_{98}, \\
& g \mapsto-E_{36}-E_{62}-E_{74}-E_{85}+E_{89} .
\end{aligned}
$$

For the second representation we define

$$
e \mapsto\left(\begin{array}{ll}
1 & 1 \\
0 & 1
\end{array}\right), \quad f \mapsto\left(\begin{array}{ll}
0 & 0 \\
1 & 0
\end{array}\right), \quad g \mapsto\left(\begin{array}{ll}
0 & 1 \\
0 & 0
\end{array}\right)
$$

One verifies directly that these are indeed representations of $\mathfrak{e c}_{3}$, and the direct sum of these two representations is faithful, from which the dimension is easily calculated. In fact, the first representation is simply the adjoint representation of $\mathfrak{e c}_{3}$, in the basis

$$
e, f, g,[e f],[e[e f]],[f g],[e[f g]],[e[e[f g]]],[f[e[e[f g]]]],
$$

and $\mathfrak{e c}_{3}$ has a one-dimensional center which acts nontrivially in the second representation. 
$\boldsymbol{Y}-\boldsymbol{\Delta}$ transformation of type $\boldsymbol{B}$. Let $e$ and $f$ be the generators of $\mathfrak{e b}_{2}$ as before, and denote by $u(t)=\exp (t e)$ and $v(t)=\exp (t f)$ the corresponding one-parameter subgroups. The following proposition is a type $B$ analog of the star-triangle transformation (Proposition 2.2).

Proposition 5.7. We have

$$
u\left(t_{1}\right) v\left(t_{2}\right) u\left(t_{3}\right) v\left(t_{4}\right)=v\left(p_{1}\right) u\left(p_{2}\right) v\left(p_{3}\right) u\left(p_{4}\right),
$$

where

$$
p_{1}=\frac{t_{2} t_{3}^{2} t_{4}}{\pi_{2}}, \quad p_{2}=\frac{\pi_{2}}{\pi_{1}}, \quad p_{3}=\frac{\pi_{1}^{2}}{\pi_{2}}, \quad p_{4}=\frac{t_{1} t_{2} t_{3}}{\pi_{1}},
$$

where

$$
\pi_{1}=t_{1} t_{2}+\left(t_{1}+t_{3}\right) t_{4}+t_{1} t_{2} t_{3} t_{4}, \quad \pi_{2}=t_{1}^{2} t_{2}+\left(t_{1}+t_{3}\right)^{2} t_{4}+t_{1} t_{2} t_{3} t_{4}\left(t_{1}+t_{3}\right) .
$$

Proof. Direct calculation inside $\mathrm{GL}_{2}$, using Lemma 5.4. We have

$$
u(t)=\left(\begin{array}{cc}
e^{t} & e^{t} t \\
0 & e^{t}
\end{array}\right), \quad v(t)=\left(\begin{array}{ll}
1 & 0 \\
t & 1
\end{array}\right),
$$

and both sides of the equality are equal to

$$
\left(\begin{array}{cc}
\mathfrak{e}^{t_{1}+t_{3}}\left(1+t_{3} t_{4}+t_{1}\left(t_{2}+t_{4}+t_{2} t_{3} t_{4}\right)\right) & e^{t_{1}+t_{3}}\left(t_{1}+t_{3}+t_{1} t_{2} t_{3}\right) \\
e^{t_{1}+t_{3}}\left(t_{2}+t_{4}+t_{2} t_{3} t_{4}\right) & e^{t_{1}+t_{3}}\left(1+t_{2} t_{3}\right)
\end{array}\right) .
$$

Remark. One can consider a one-parameter family of deformations of the above formulae by taking

$$
\pi_{1}=t_{1} t_{2}+\left(t_{1}+t_{3}\right) t_{4}+\tau t_{1} t_{2} t_{3} t_{4}, \quad \pi_{2}=t_{1}^{2} t_{2}+\left(t_{1}+t_{3}\right)^{2} t_{4}+\tau t_{1} t_{2} t_{3} t_{4}\left(t_{1}+t_{3}\right) .
$$

At $\tau=0$ this specializes to the transformation found in [Berenstein and Zelevinsky 1997, Theorem 3.1]. Furthermore, just like that transformation, this deformation is given by positive rational formulae.

\section{References}

[Berenstein and Zelevinsky 1997] A. Berenstein and A. Zelevinsky, "Total positivity in Schubert varieties”, Comment. Math. Helv. 72:1 (1997), 128-166. MR 99g:14064 Zbl 0891.20030

[CdVGV 1996] Y. Colin de Verdière, I. Gitler, and D. Vertigan, "Réseaux électriques planaires, II", Comment. Math. Helv. 71:1 (1996), 144-167. MR 98a:05054 Zbl 0853.05074

[CIM 1998] E. B. Curtis, D. Ingerman, and J. A. Morrow, "Circular planar graphs and resistor networks”, Linear Algebra Appl. 283:1-3 (1998), 115-150. MR 99k:05096 Zbl 0931.05051

[Fomin and Reading 2007] S. Fomin and N. Reading, "Root systems and generalized associahedra", pp. 63-131 in Geometric combinatorics (Park City, UT, 2004), edited by E. Miller et al., IAS/Park City Math. Ser. 13, Amer. Math. Soc., Providence, RI, 2007. MR 2383126 Zbl 1147.52005

[Fulton and Harris 1991] W. Fulton and J. Harris, Representation theory, Graduate Texts in Mathematics 129, Springer, New York, 1991. MR 93a:20069 Zbl 0744.22001 
[Humphreys 1990] J. E. Humphreys, Reflection groups and Coxeter groups, Cambridge Studies in Advanced Mathematics 29, Cambridge University Press, 1990. MR 92h:20002 Zbl 0725.20028

[Kennelly 1899] A. E. Kennelly, "Equivalence of triangles and stars in conducting networks", Electrical World and Engineer 34:12 (1899), 413-414.

[Lusztig 1994] G. Lusztig, “Total positivity in reductive groups”, pp. 531-568 in Lie theory and geometry, edited by J.-L. Brylinski et al., Progr. Math. 123, Birkhäuser, Boston, 1994. MR 96m:20071 Zbl 0845.20034

Communicated by Georgia Benkart

Received 2014-05-19 Revised 2015-04-09 Accepted 2015-06-11

tfylam@umich.edu

Department of Mathematics, University of Michigan, 530 Church St., Ann Arbor, MI 48109, United States

ppylyavs@umn.edu

Department of Mathematics, University of Minnesota, 206 Church St. SE, Minneapolis, MN 55455, United States 


\section{Algebra \& Number Theory}

msp.org/ant

\section{EDITORS}

MANAGING EDITOR

Bjorn Poonen

Massachusetts Institute of Technology

Cambridge, USA

\author{
EDITORIAL BOARD CHAIR \\ David Eisenbud \\ University of California \\ Berkeley, USA
}

BOARD OF EDITORS

Georgia Benkart

Dave Benson

Richard E. Borcherds

John H. Coates

J-L. Colliot-Thélène

Brian D. Conrad

Hélène Esnault

Hubert Flenner

Sergey Fomin

Edward Frenkel

Andrew Granville

Joseph Gubeladze

Roger Heath-Brown

Craig Huneke

Kiran S. Kedlaya

János Kollár

Yuri Manin

Philippe Michel
University of Wisconsin, Madison, USA

University of Aberdeen, Scotland

University of California, Berkeley, USA

University of Cambridge, UK

CNRS, Université Paris-Sud, France

Stanford University, USA

Freie Universität Berlin, Germany

Ruhr-Universität, Germany

University of Michigan, USA

University of California, Berkeley, USA

Université de Montréal, Canada

San Francisco State University, USA

Oxford University, UK

University of Virginia, USA

Univ. of California, San Diego, USA

Princeton University, USA

Northwestern University, USA

École Polytechnique Fédérale de Lausanne
Susan Montgomery

Shigefumi Mori

Raman Parimala

Jonathan Pila

Anand Pillay

Victor Reiner

Peter Sarnak

Joseph H. Silverman

Michael Singer

Vasudevan Srinivas

J. Toby Stafford

Ravi Vakil

Michel van den Bergh

Marie-France Vignéras

Kei-Ichi Watanabe

Efim Zelmanov

Shou-Wu Zhang
University of Southern California, USA

RIMS, Kyoto University, Japan

Emory University, USA

University of Oxford, UK

University of Notre Dame, USA

University of Minnesota, USA

Princeton University, USA

Brown University, USA

North Carolina State University, USA

Tata Inst. of Fund. Research, India

University of Michigan, USA

Stanford University, USA

Hasselt University, Belgium

Université Paris VII, France

Nihon University, Japan

University of California, San Diego, USA

Princeton University, USA

\section{PRODUCTION}

production@msp.org

Silvio Levy, Scientific Editor

See inside back cover or msp.org/ant for submission instructions.

The subscription price for 2015 is US $\$ 255 /$ year for the electronic version, and $\$ 440 /$ year ( $+\$ 55$, if shipping outside the US) for print and electronic. Subscriptions, requests for back issues and changes of subscribers address should be sent to MSP.

Algebra \& Number Theory (ISSN 1944-7833 electronic, 1937-0652 printed) at Mathematical Sciences Publishers, 798 Evans Hall \#3840, c/o University of California, Berkeley, CA 94720-3840 is published continuously online. Periodical rate postage paid at Berkeley, CA 94704, and additional mailing offices.

ANT peer review and production are managed by EditFLOW ${ }^{\circledR}$ from MSP.

\section{PUBLISHED BY}

- mathematical sciences publishers

nonprofit scientific publishing

http://msp.org/

(C) 2015 Mathematical Sciences Publishers 


\section{Algebra \& Number Theory}

Volume $9 \quad$ No. $6 \quad 2015$

Bivariant algebraic cobordism

José LUIS GONZÁLEZ and KALLE KARU

Schubert decompositions for quiver Grassmannians of tree modules OLIVER LORSCHEID

Noncommutative geometry and Painlevé equations

ANDREI OKOUNKOV and ERIC RAINS

Electrical networks and Lie theory

ThOMas Lam and Pavlo PylyavskyY

The Kac-Wakimoto character formula for the general linear Lie superalgebra Michael Chmutov, Crystal Hoyt and ShifRa Reif

Effective Matsusaka's theorem for surfaces in characteristic $p$

Gabriele Di Cerbo and ANDREA FANELli

Adams operations and Galois structure

GEORGIOS PAPPAS 